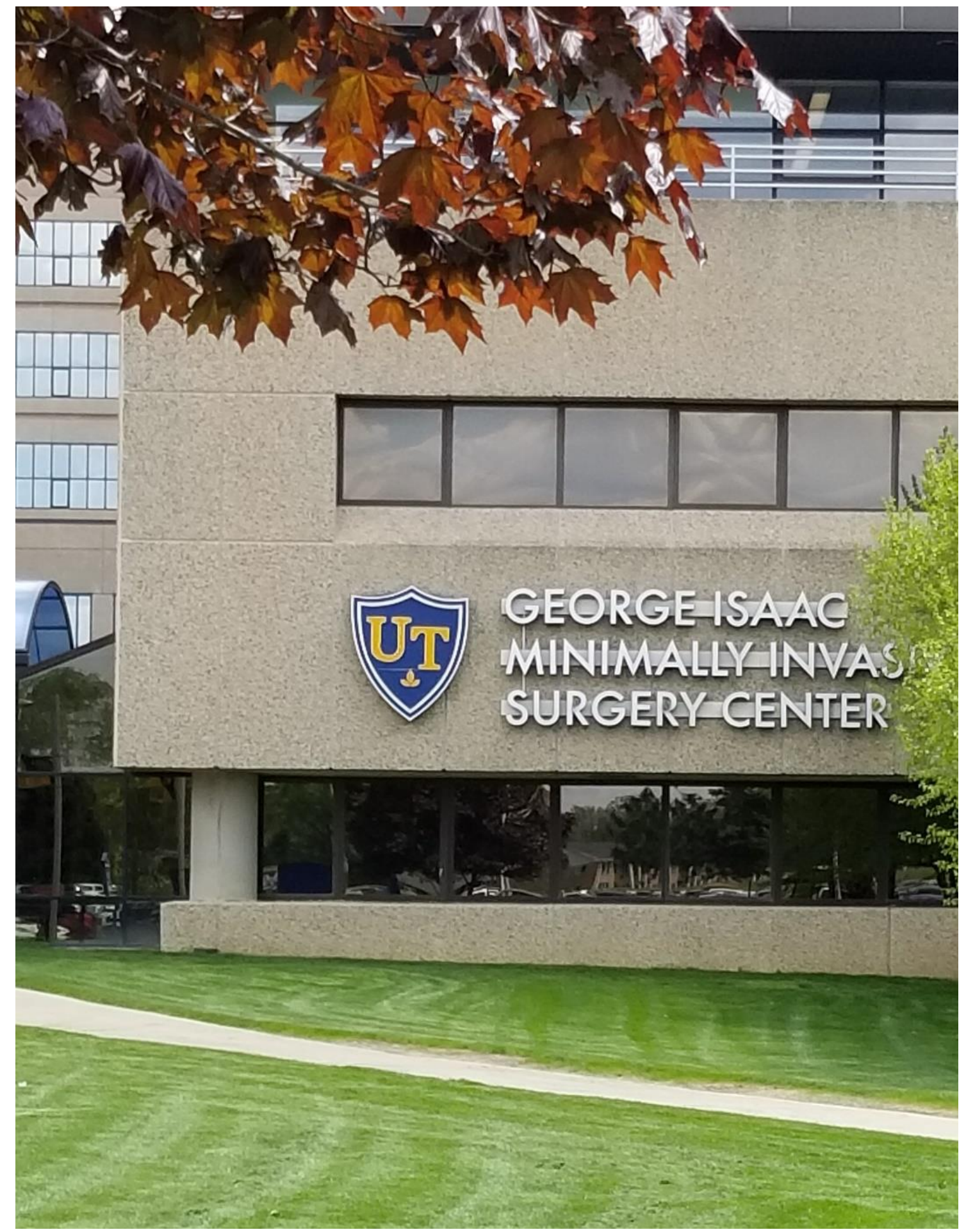




\title{
Boondoggled: A Case Report of Arts and Crafts Gone Wrong
}

\author{
David Fumo $^{a, 1}$ Damian Garcher $^{a}$ Alice Bonnell $^{a, b}$ and Puneet Sindhwani ${ }^{a}$
}

${ }^{a}$ Department of Urology, The University of Toledo, Health Science Campus, Toledo, USA, and ${ }^{b}$ Mercy Health Saint Vincent Medical Center, Toledo, OH, USA

\begin{abstract}
Self-insertion of foreign bodies into the urinary tract is a notuncommon urological problem with significant psychosocial implications. A 12-year-old male presented to the ED when his father noted blood in the patient's urine. During evaluation, the patient admitted to inserting Boondoggle, a plastic arts and crafts string, into his urethra 1 week prior. He admitted to performing this activity on multiple prior occasions. He subsequently developed worsening frequency, urgency, dysuria, hematuria, and suprapubic pain. Bladder ultrasound and abdominal $x$-ray were obtained which demonstrated a large amount of hyperdense material coiled in the bladder. He was brought to the operating room the following day for removal of the foreign body. After failed attempts at endoscopic retrieval, 34 feet of plastic string was removed via an open approach. The bladder was closed and a Foley catheter was left in place. He was discharged on postoperative day 2 on culture specific antibiotics after a successful void trial. Urethral masturbation has been associated with psychiatric disorders, fetishism, and sadomasochistic tendencies. For the urologist, it is critical to recognize the mental health issues portended by urethral foreign body insertion, and take the appropriate steps to address the underlying problem.
\end{abstract}

genitourinary foreign body | bladder | urethra urinary injury | polyembolokoilamania endoscopy |

$\mathbf{R}$ eports of genitourinary foreign bodies (GUFB) are abundant in the literature. The etiology of GUFB can range from iatrogenic, the result of penetrating trauma, migration from adjacent organs, or self-insertion (1-8). Self-insertion, by far the most commonly reported etiology in the literature, has been implicated in a number of underlying psychosocial, developmental, and masochistic disorders. The need for psychiatric referral is commonly discussed, even when no known psychiatric diagnosis exists (9-11). The range of objects reportedly found in the genitourinary tract is truly astounding, and appears to be limited only by the caliber of the urethra and the creativity of the patient (12).

Symptoms of GUFB are typically those of the lower urinary tract, including dysuria, hematuria, suprapubic tenderness, or retention $(13,14)$. Diagnosis is often made via patient history and symptomatology. However, this can be difficult as shame and humiliation often deters patients from being completely forthright about the preceding event. Imaging modalities including ultrasound, plain x-ray, and computed tomography (CT) have been used to aid in or confirm diagnosis, as well as to plan removal strategies. Surgical intervention is typically required, and should proceed stepwise from least to most invasive modality necessary $(12,15)$.

While removal of GUFB may require a degree of creativity on the part of the physician, the instruments and surgical techniques reported are generally not outside the skillset of the average urologist. However, the overall management of these patients is not as straightforward. The focus should be on preventing recurrence, as GUFB self-insertion is usually not an isolated event (16-18). Particular attention should be paid to addressing the underlying motivations for this erratic behavior. At the very least this should include extensive education regarding the dangers of such activities. Psychiatric referral should almost always be considered to address the psychosocial disturbances that lead to this behavior.

\section{Case report}

Patient information. Age: 12 years, gender: male, ethnicity: caucasian. Related medical problems: urinary tract infection, hematuria, genitourinary foreign body.

Objective. To discuss psychosocial implications of genitourinary foreign body self-insert.

Case. A 12-year-old male presented to the ED when his father noted blood in the patient's urine. When confronted, the patient admitted to inserting Boondoggle, a plastic arts and crafts string, into his urethra 1 week prior. He admitted to performing this activity on multiple prior occasions because "it feels like sex". On this occasion the string was unable to be retrieved despite multiple extraction attempts with a Q-tip. He subsequently developed worsening frequency, urgency, dysuria, hematuria, and suprapubic pain. Bladder ultrasound and abdominal x-ray were obtained which demonstrated a large amount of hyperdense material coiled in the bladder (Fig. 1). Past medical history was significant for depression and attention deficit disorder. There was also a question of autism spectrum disorder. Two months prior he had been in a motor vehicle collision resulting in multiple facial lacerations with significant scarring. His parents were also recently divorced resulting in a complicated and fluctuating home environment.

Afebrile with no evidence of systemic infection, he was admitted, started on antibiotics, and brought to the operating room the following day for removal of the foreign body. On cystoscopy the bladder was severely inflamed and filled with neon green string (Fig. 1B). Attempts at transurethral removal were quickly abandoned as the string became knotted. The bladder was accessed via a pfannenstiel incision. The knotted ball of boondoggle was carefully extracted which, when unraveled, consisted of a single strand 34 feet in length (Fig. 1 C-D). The bladder was irrigated thoroughly, closed, and a Foley catheter was left in place. He was discharged on postoperative day two on culture specific antibiotics after a success-

All authors contributed to this paper. ${ }^{1}$ To whom correspondence should be sent: David.Fumo@utoledo.edu

The authors declare no conflict of interest. Submitted: May/07/2019, published: July/19/2019. Freely available online through the UTJMS open access option 
ful void trial. The patient was lost to follow up despite psychiatric referral and social services involvement.

\section{Discussion}

The etiology of GUFB is varied. Iatrogenic foreign bodies are a particularly sensitive issue with both medical and legal ramifications. Rafique et al. reported 16 cases of GUFB, of which 7 were iatrogenic. Foreign bodies included gauze sponges, a piece of foley balloon, and the Teflon beak of a resectoscope (14). Multiple other reports of iatrogenic GUFB include sutures, mesh, migrated slings and sphincters, and silastic stents $(1,3,19-22)$. In females, objects such as tampons, thermometers, and incontinence plugs can inadvertently be lost in the bladder following incorrect insertion (4, $6,14)$. Kamil et al. reported a case of a foreign body found in the bladder of a 48-year-old female, which was accidentally inserted by her partner during a consensual sexual act (5).

Polyembolokoilamania, a form of paraphilia, is a drastically more complex problem than the cases of foreign bodies accidentally inserted into the GU tract mentioned above. In a review of the psychological profiles of multiple cases, Kenny postulated that a serendipitously discovered pleasurable stimulation of the urethral mucosa can be an inciting event, leading to repetition with various objects of unrecognized danger (16). The prevalence of such behavior is unknown as patients only come to attention when inserted objects are unable to be retrieved. While these objects are often removed without significant incident, serious complications have been reported, including urethral stricture, bladder perforation, and even complete urethral evulsion $(2,7,8,15,23,24)$. In 1988 Wyman reported the development of squamous cell carcinoma of the bladder associated with an intravesical foreign body (25). While uncommon, fatalities have been reported (26).

It has been postulated that traumatic events, underlying psychiatric disorders, or unusually strong libidinal drives could modify normal psychosexual drives leading to a fixation or regression to a urethral stage of eroticism1 $(1,12,18)$. The prevailing themes present in the psychiatric evaluation of GUFB self-inserters include chaotic and unstable home lives in childhood, psychosexual development disorders, sadomasochistic tendencies, and self-injurious behavior $(10,16)$. In the case presented, the patient displays multiple red flags for underlying psychosexual derangements. With a a history of recent trauma and an unstable home life, regression to urethral libidinal eroticism in a pre-pubertal male should be perceived as a sentinel event warranting psychiatric evaluation.

1. Athanasopoulos A, Liatsikos EN, Perimenis P, and Barbalias GA (2002) Delayed suture intravesical migration as a complication of a Stamey endoscopic bladder neck suspension.Int Urol Nephrol 34(1):5-7.

2. Bantis A, et al. (2010) Perforation of the urinary bladder caused by transurethral insertion of a pencil for the purpose of masturbation in a 29 -year-old female. Case Rep Med 2010.

3. Bartoletti R, Gacci M, Travaglini F, Sarti E, and Selli C (2000) Intravesical migration of AMS $\mathbf{8 0 0}$ artificial urinary sphincter and stone formation in a patient who underwent radical prostatectomy. Urol Int 64(3):167-168.

4. Hoscan MB, Kosar A, Gumustas U, and Guney M (2006) Intravesical migration of intrauterine device resulting in pregnancy. Int $J$ Urol 13(3):301-302.

5. Kamil A, Cozman C, Aslam A, Nusrat N, and Jaffry S (2018) Valentine's day misadventure: Missing vagina for urethra. Urol Case Rep 18:89-90.

6. Scriven JM and Patterson JE (1995) Extraction of an intravesical thermometer using a flexible cystoscope. Br J Urol 76(6):815.

7. Stamatiou $K$ and Moschouris $H$ (2016) A rubber tube in the bladder as a complication of autoerotic stimulation of the urethra. Arch Ital Urol Androl 88(3):239-240.

8. Trehan RK, Haroon A, Memon S, and Turner D (2007) Successful removal of a telephone cable, a foreign body through the urethra into the bladder: a case report. J Med Case Rep 1:153.
Unfortunately, as is commonly the case, patient and family compliance with recommendations was not followed. Social workers have subsequently been involved with no resolution.

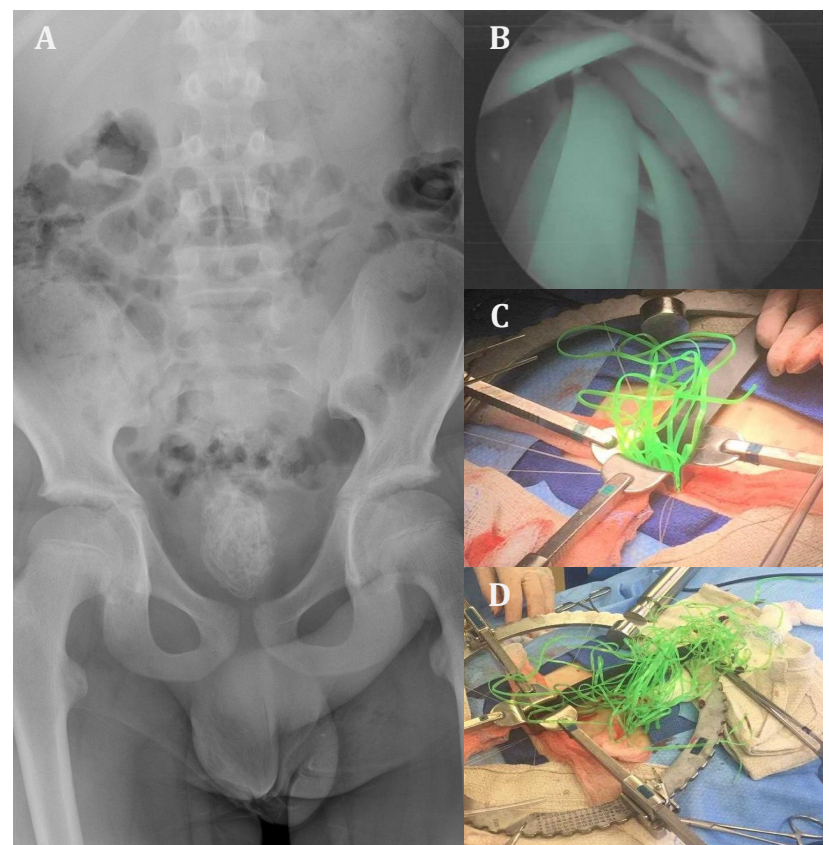

Figure 1. A-abdominal X-Ray with hyperdense foreign body visible in the bladder; $B$ - endoscopic view of coiled foreign body within the bladder; $C$ - open surgical removal of foreign body, mid-extraction; $D$ - open surgical removal of foreign body, post-extraction.

Conclusion. GUFB insertion, particularly in children, is frequently the presenting symptom of an underlying psychosocial/psychosexual disorder. It should be viewed as a harbinger of future antisocial and self-injurious behavior. In children, urethral foreign body insertion is more commonly associated with mischief or curiosity. This case details an alarming sexual act in a pre-pubescent adolescent, which represents an early deviation in psychosexual development. For the urologist, it is critical to recognize the mental health issues portended by urethral foreign body insertion, and take the appropriate steps to address the underlying problem.

9. Boscolo-Berto R, lafrate $M$, and Viel $G$ (2010) Forensic implications in selfinsertion of urethral foreign bodies. Can J Urol 17(1):5026-5027.

10. Costa G, et al. (1993) Self-introduction of foreign bodies into the urethra: a multidisciplinary problem. Int Urol Nephrol 25(1):77-81.

11. Unruh BT, Nejad SH, Stern TW, and Stern TA (2012) Insertion of foreign bodies (polyembolokoilamania): underpinnings and management strategies. Prim Care Companion CNS Disord 14(1).

12. van Ophoven $A$ and deKernion JB (2000) Clinical management of foreign bodies of the genitourinary tract. $J$ Urol 164(2):274-287.

13. Palmer CJ, et al. (2016) Urethral Foreign Bodies: Clinical Presentation and Management. Urology 97:257-260.

14. Rafique $M(2008)$ Intravesical foreign bodies: review and current management strategies. Urol J 5(4):223-231.

15. Datta $B$, Ghosh $M$, and Biswas $S$ (2011) Foreign bodies in urinary bladders. Saudi $\mathrm{J}$ Kidney Dis Transpl 22(2):302-305.

16. Kenney RD (1988) Adolescent males who insert genitourinary foreign bodies: is psychiatric referral required? Urology 32(2):127-129.

17. Mitchell WM (1968) Self-insertion of urethral foreign bodies. Psychiatr $Q$ 42(3):479-486.

18. Wise TN (1982) Urethral manipulation: an unusual paraphilia. J Sex Marital Ther $8(3): 222-227$. 
19. Bodenbach M, Bschleipfer T, Stoschek M, Beckert R, and Sparwasser C (2002) [Intravesical migration of a polypropylene mesh implant 3 years after laparoscopic transperitoneal hernioplasty]. Urologe $A$ 41(4):366-368.

20. Hutton KA and Huddart SN (1999) Percutaneous retrieval of an intravesical foreign body using direct transurethral visualization: a technique applicable to small children. BJU Int 83(3):337-338.

21. Nabi G, Hemal AK, and Khaitan A (2001) Endoscopic management of an unusual foreign body in the urinary bladder leading to intractable symptoms. Int Uro Nephrol 33(2):351-352.

22. Rosenblatt P, Pulliam S, Edwards R, and Boyles SH (2005) Suprapubically assisted operative cystoscopy in the management of intravesical TVT synthetic mesh segments. Int Urogynecol J Pelvic Floor Dysfunct 16(6):509-511.
23. Chan G, Mamut A, Tatzel S, and Welk B (2016) An unusual case of polyembolokoilamania: Urethral avulsion from foreign object use during sexual gratification. Can Urol Assoc J 10(5-6):E181-E183.

24. Rahman NU, Elliott SP, and McAninch JW (2004) Self-inflicted male urethral foreign body insertion: endoscopic management and complications. BJU Int 94(7):1051-1053.

25. Wyman A and Kinder RB (1988) Squamous cell carcinoma of the bladder associated with intrapelvic foreign bodies. Br J Urol 61(5):460.

26. Byard RW, Eitzen DA, and James $R(2000)$ Unusual fatal mechanisms in nonasphyxial autoerotic death. Am J Forensic Med Pathol 21(1):65-68. 